\title{
High cytoplasmic YAP1 expression predicts a poor prognosis in patients with colorectal cancer
}

\author{
Tianqi Dong ${ }^{1}$, Yuncang Yuan ${ }^{1}$, Xudong Xiang ${ }^{2}$, Shuping Sang ${ }^{1}$, Hao Shen ${ }^{1}$, Lei Wang ${ }^{1}$, Chunyan Yang $^{1}$, Li \\ Fangfang ${ }^{1}$, Hongliang Li ${ }^{\text {Corresp., }}$, Shangyong Zheng ${ }^{\text {Corresp. } 1}$ \\ 1 school of medicine, Yunnan University, Kunming, Yunnan, China \\ 2 Department of Thoracic Surgery, Third Affiliated Hospital of Kunming Medical University, Kunming, Yunnan, China \\ Corresponding Authors: Hongliang Li, Shangyong Zheng \\ Email address: lihongliang@ynu.edu.cn, shangyong@ynu.edu.cn
}

Purpose: Yes associated protein 1 (YAP1), which is a standout amongst the most essential effectors of the Hippo pathway, assumes a vital part in a few kinds of cancer. However, whether YAP1 is an oncogene in CRC (colorectal cancer) remains controversial, and the association between the subcellular localization of YAP1 and clinical implications in CRC remains unknown. Patients and methods: In this study, we investigated the subcellular localization of YAP1 in CRC cells by immunohistochemistry and then associate these findings with clinical information in a large CRC cohort with 922 CRC patients. Results: The results show that CRC tissues has a significant higher expression of cytoplasmic YAP1 compared to adjacent normal tissues (all $P<0.001$ ). Cytoplasmic YAP1 expression was significantly associated with the number of lymph nodes removed and differentiation grade (all $P<0.001$ ). Furthermore, after correcting confounding variables, for example, TNM stage and differentiation grade, the multivariate Cox analysis confirmed cytoplasmic YAP1-high subgroup had a significant shorter DFS(HR=3.255; 95\% $\mathrm{Cl}=2.290-4.627$; $P<0.001)$ and $\mathrm{DSS}(\mathrm{HR}=4.049 ; 95 \% \mathrm{Cl}=2.400-6.830 ; P<0.001)$ than cytoplasmic YAP1-low subgroup. High cytoplasmic YAP1 expression is associated with a worse survival in stage III CRC patients who received chemotherapy. Conclusion: Cytoplasmic YAP1 could be could be utilized as a prognosis factor in CRC patients, and may be an indicator of whether certain patients population could benefit from postoperative chemotherapy. Key words: colorectal cancer; YAP1; subcellular localization; immunohistochemistry; prognosis; chemotherapy 
1 Title: High cytoplasmic YAP1 expression predicts a poor prognosis in patients with colorectal 2 cancer.

\section{Authors:}

5 Tianqi Dong ${ }^{1}$, Yuncang Yuan ${ }^{1}$, Xudong Xiang ${ }^{2}$, Shuping Sang ${ }^{1}$, Hao Shen ${ }^{1}$, Lei Wang ${ }^{1}$,

6 Chunyan Yang ${ }^{1}$, Li Fangfang ${ }^{1}$, Hongliang $\mathrm{Li}^{1}{ }^{1, *}$ and Shangyong Zheng ${ }^{1, *}$

7

8 Authors' Affiliations:

9 'School of Medicine, Yunnan University, No. 2 North Cuihu Road, Kunming 650091, P. R. 10 China.

11 2Department of Thoracic Surgery, Third Affiliated Hospital of Kunming Medical University, No. 12519 Kunzhou Road, Kunming 650118, P. R. China.

13

14 "Correspondence to: Dr. Hongliang Li, School of Medicine, Yunnan University, No. 2 North 15 Cuihu Road, Kunming 650091, P. R. China. Email: lihongliang@ynu.edu.cn. Tel.: +86-87116 65034358; Dr. Shangyong Zheng, School of Medicine, Yunnan University, No. 2 North Cuihu 17 Road, Kunming 650091, P. R. China. Email: shangyong@ynu.edu.cn. Tel.: +86-871-65034358. 
18 Abstract

19 Purpose: Yes associated protein 1 (YAP1), which is a standout amongst the most essential 20 effectors of the Hippo pathway, assumes a vital part in a few kinds of cancer. However, whether

21 YAP1 is an oncogene in CRC (colorectal cancer) remains controversial, and the association

22 between the subcellular localization of YAP1 and clinical implications in CRC remains 23 unknown.

24 Patients and methods: In this study, we investigated the subcellular localization of YAP1 in 25 CRC cells by immunohistochemistry and then associate these findings with clinical information 26 in a large CRC cohort with $922 \mathrm{CRC}$ patients.

27 Results: The results show that $\mathrm{CRC}$ tissues has a significant higher expression of cytoplasmic 28 YAP1 compared to adjacent normal tissues (all $P<0.001$ ). Cytoplasmic YAP1 expression was 29 significantly associated with the number of lymph nodes removed and differentiation grade (all $P$ $30<0.001)$. Furthermore, after correcting confounding variables, for example, TNM stage and 31 differentiation grade, the multivariate Cox analysis confirmed cytoplasmic YAP1-high subgroup 32 had a significant shorter DFS(HR=3.255; 95\% $\mathrm{CI}=2.290-4.627 ; P<0.001)$ and $\mathrm{DSS}(\mathrm{HR}=4.049$; $3395 \% \mathrm{CI}=2.400-6.830 ; P<0.001)$ than cytoplasmic YAP1-low subgroup. High cytoplasmic YAP1 34 expression is associated with a worse survival in stage III CRC patients who received 35 chemotherapy.

36 Conclusion: Cytoplasmic YAP1 could be could be utilized as a prognosis factor in CRC 37 patients, and may be an indicator of whether certain patients population could benefit from 38 postoperative chemotherapy.

39 Key words: colorectal cancer; YAP1; subcellular localization; immunohistochemistry; 40 prognosis; chemotherapy 


\section{Introduction}

42

The prevalence of CRC ranks third among all cancers in male and second in female (Torre et al. 2015), and CRC has the third highest mortality rate among all cancers (Zeng et al. 2014). Surgical resection combined with chemotherapy remains the mainstay of treatment for CRC, in any case, numerous patients will progress to metastatic CRC and develop resistance to chemotherapeutic drugs (Fisher et al. 2015), because signs or symptoms diagnose CRC usually appear in advanced phases (Binefa et al. 2014). Even if some patients are diagnosed with CRC and undergo surgery at an early stage, $20 \%-30 \%$ of these patients will relapse within five years. (Hardingham et al. 2015). Current CRC treatment regimen is heterogenous for patients, even for patients with the same TNM stage (Nagtegaal et al. 2012), in any case, the indication for treatment should be assessed on an individual basis by considering the risk factors of relapse (Marin et al. 2012). Currently, the only effective marker for the CRC prognosis and appropriate chemotherapy selection is microsatellite instability (MSI) (Hemminki et al. 2000; Popat et al. 2005), however, MSI as a CRC marker has not been applied clinically. Therefore, there is an urgent need for new biomarkers to assess the prognosis of CRC patients before and after treatment.

YAP1 is a standout amongst the most essential effectors of the Hippo pathway, which is a critical pathway regulating cell proliferation, apoptosis, and organ growth (Justice et al. 1995). Several studies have shown that YAP1 is an oncogene highly express in numerous cancer types including bladder cancer (Liu et al. 2013), breast cancer (Kim et al. 2014), gastric cancer (Kang et al. 2011), hepatocellular cancer (Xu et al. 2009), nonsmall-cell lung cancer (Wang et al. 2010), and CRC (Wang et al. 2013a; Xu et al. 2009) that associate with tumor progression and poor prognosis. On the contrary, abundant literature suggested that YAP1 is a tumor suppressor gene and nuclear expression is reduced in different cancers, such as breast cancer (Matallanas et al. 2007; Yu et al. 2013; Yuan et al. 2008), head and neck cancers (Ehsanian et al. 2010), hematological cancers (Cottini et al. 2014), and CRC (Levy et al. 2007). These paradoxical reports remind us that the role of YAP1 in cancer is controversial, and it is crucial to make it clear the relationship between YAP1 expression and its clinical relevance in CRC. In addition, the nuclear overexpression of YAP1 is associate with poor survival in gastric cancer (Kang et al. 2011), actually, previous researches suggest that subcellular localization of proteins is associated with functions associated of tumorigenesis and tumor progression (Garcia et al. 2005; Lobo et al. 2009; Vaquero et al. 2017), a few studies suggested that YAP1 overexpression is associate with poor survival in CRC (Wang et al. 2013a; Wang et al. 2013b; Yang et al. 2018), however, the association between subcellular localization of YAP1 and clinical significance in CRC has been 
75 largely ignored. Thus, the prognostic significance of YAP1 in CRC needs further investigation.

On the basis of these considerations, we explore the the subcellular localization of YAP1 in $922 \mathrm{CRC}$ colorectal tissues by immunohistochemistry performed on tissue microarrays (TMAs), and investigate the association between subcellular localization of YAP1 and patient's survival. This study may provide deeper insights to understand the role of YAP1 in prognosis and treatment of CRC.

\section{Materials and Methods}

\section{Bioinformatics analysis}

All available related mRNA expression profiles of YAP1 in CRC tissues were downloaded from the TCGA database and normalized by EDASeq package which can take gene length and GC-content into account. The microarray expression profiles of eight datasets (GSE8671, GSE37364, GSE41258, GSE23878, GSE22598, GSE9348, GSE81582, and GSE77955) associated with CRC tissues were randomly downloaded from the Gene Expression Omnibus (GEO) database, , then the mRNA expression profiles of YAP1 in different pathological feature CRC tissues were extracted from the microarray expression profiles and compared by independent sample $t$-tests or paired sample $t$-tests.

\section{Patient characteristics}

Between January 2001 and November 2011, we collected a total of 1067 CRC tissue samples donated by 929 patients who underwent surgery at Yunnan Cancer Hospital\&Third Affiliated Hospital of Kunming Medical University (Kunming, P. R. China), then seven tissue microarrays (TMAs) were constructed with these samples by a biotechnology company (Outdo Biotech, Shanghai, P. R. China) as reported previously (Pan et al. 2015). The core on the TMA is $1.2 \mathrm{~mm}$ in diameter and one core represents a sample, and there are 70 normal samples, 33 adenoma samples, 949 primary cancer samples, and 15 metastatic cancer samples on TMAs.

Pathological diagnosis and staging of all patients based on the 7th American Joint Committee on Cancer Staging System, the clinic characteristics of all patients, including age, sex, disease 02 location, TNM stage, differentiation grade, number of resected lymph nodes, chemotherapy 3 (FOLFOX regimen), serum carcinoembryonic antigen (CEA), and carbohydrate antigen 19-9 04 (CA19-9) were summarized in Table 1. This study was approved by The Committee on Human 05 Subject Research and Ethics, Yunnan University (approval number: ynucare20200347). All 
patients signed a written informed consent for using their tissues for research purpose.

\section{Immunohistochemistry (IHC)}

$108 \quad$ IHC is performed on $4 \mu \mathrm{m}$ thick array slides. Specifically, the array slides were primarily 109 immersed into the citrate solution ( $\mathrm{pH} \mathrm{6.0)}$ and boil for 5 minutes for antigen retrieval, then 110 incubated with 10\% goat serum (SL038; Solarbio, Beijing, P. R. China) for 30 minutes at room 111 temperature to block non-specific binding, subsequently, Mouse antihuman YAP1 primary 112 monoclonal antibody (1:100, sc-376830; Santa Cruz Biotechnology, Santa Cruz, CA, USA) was

113 used to incubate array slides at $4^{\circ} \mathrm{C}$ overnight, and secondary antibody included in the

114 Maxvision $^{\text {TM }}$ HRP-Polymer Anti-Mouse IHC Kit (KIT-5920; Maxvision, P. R. China) was used

115 to incubate array slides 10 mins at room temperature. All array slides performed IHC

116 simultaneously and strictly comply with the standard protocol.

\section{Quantitative evaluation of immunostaining}

118 Aperio ScanScope (Aperio Technologies, Vista, CA, USA) was used to digitally scan the 119 stained TMA slides, then the scan image can be used for Quantitative evaluation of 120 immunostaining, the YAP1 protein expression level was quantified by H-score method as 121 reported previously (Detre et al. 1995). Specifically, the staining intensity in the epithelial cell 122 was scored as $0,1,2$, or 3 corresponding to the presence of negative, weak, intermediate, and 123 strong brown staining, respectively, then the number of cells stained at each intensity was 124 counted. The H-score is the multiplication of the proportion of positive cells and the 125 corresponding staining intensity score $(0,1,2$ or 3$)$, thus an $\mathrm{H}$-score between 0 and 300 was 126 obtained. The quantitative evaluation of immunostaining was performed separately by two co127 authors who were blinded to the clinicopathological information, and the scores were averaged.

\section{Follow-up and patients}

919 CRC patients' follow-up information was collected using a standard methods reported 130 previously (Pan et al. 2015). Disease-free survival (DFS) was defined as the number of months 131 from the first treatment to the first relapse. Disease-specific survival (DSS) as the number of 132 months from the first treatment to the date of death due to $\mathrm{CRC}$. The patients were divided into 133 two subgroups (cytoplasmic YAP1 high vs. cytoplasmic YAP1 low, nuclear YAP1 high vs. nuclear

134 YAP1 low, or YAP1 NCR high vs. YAP1 NCR low) by the optimal cut-off values for maximum 135 discrimination in survival difference, the cut-off values were determined by the maxstat R package 
136 in R 3.2.0.

\section{Statistical analysis}

138 Clinicopathological characteristics of all CRC patients were summarized in related tables, in 139 which continuous variables were tested by two-sample Student $t$-tests, and categorical variables 140 were tested by Pearson Chi-squared tests, the TNM stage and differentiation grade were test by

141 Wilcoxon-Mann-Whitney tests. The DSS and DFS of patient's subgroups were compared by

142 Kaplan-Meier analysis with log-rank test to examine the difference. All factors were determined

143 their independence of the prognostic value by Univariate and multivariate Cox regression

144 analyses (Further evaluation of meaningful prognostic factors in univariate analysis in

145 multivariate analysis). All statistical analyses were conducted by SPSS 21 for Windows (IBM

146 Inc., Armonk, NY, USA) and it was considered statistically significant if $P<0.05$.

\section{Results}

\section{Differences in YAP1 expression between CRC tissues and adjacent normal tissues.}

149 To analyze the expression pattern of YAP1 in CRC tissues, we firstly utilized the datasets 150 from public database, the results showed that in one TCGA dataset and three GEO datasets, 151 YAP1 mRNA expression level was consistently significantly elevated in CRC tissues compared 152 with the adjacent normal tissue (all $P<0.01$; Fig. 1A-D), the other five GEO datasets also show 153 the same results (all $P<0.01$; Fig. S1A). We subsequently investigated the expression pattern of 154 YAP1 by IHC method in $997 \mathrm{CRC}$ and 70 adjacent normal tissue samples which derive from 155 patients who underwent surgery at Yunnan Cancer Hospital. The positive immunostaining results 156 from YAP1 predominantly occurred in the cytoplasm and nucleus of colorectal epithelial cells 157 (Fig. 1H-M), whereas the staining was negative or weak in mesenchymal cells (Fig. 1H-M). We 158 calculated the H-score of cytoplasmic YAP1 and nuclear YAP1 independently, then the YAP1 159 NCR (nuclear/cytoplasmic ratio) was calculated, and there is a very weak positive correlation 160 between cytoplasmic H-score and nuclear YAP1 H-score (Fig. S1B). the results show that 161 cytoplasmic YAP1 expression was significantly elevated in CRC tissues compared with the 162 adjacent normal tissues (all $P<0.001$; Fig. 1E), and nuclear YAP1 expression was significantly 163 elevated in primary cancer tissues compared with the adjacent normal tissues $(P<0.0001$; Fig. $1641 \mathrm{~F})$, but the expression of nuclear YAP1 in adenomas and metastasis CRC tissues have no 165 significant differences with the adjacent normal tissues (Fig. 1F), we also found YAP1 NCR 166 (Nuclear/Cytoplasmic Ratio) gradually decrease in adjacent normal tissues, adenomas, primary 
167 cancers, and metastatic $\mathrm{CRC}$ (all $\mathrm{P}<0.05$; Fig. 1G). The results above indicated that the increased

168 cytoplasmic YAP1 expression may be associated with the progression of CRC.

\section{Associations between YAP1 expression and CRC patients' clinicopathological}

170

171

172

173

174

175

176

177

178

179

180

181

182

183

184

185

186

187

188

189

190

191

192

193

194

195

196

197

198 characteristics

To obtain further information, we analyzed the association between cytoplasmic YAP1 expression levels or YAP1 NCR and CRC patients' clinicopathological characteristics. We found that the expression of cytoplasmic YAP1 protein was significantly higher in poorly+moderate grades than that in the well grade $(P<0.001$; Fig. S1C), and YAP1 NCR was significantly lower in poorly+moderate grades than that in the well grade $(P<0.001$; Fig. $\mathrm{S} 1 \mathrm{E})$, but there is no significant differences between poorly+moderate grades and well grade in the expression of nuclear YAP1 protein (Fig. S1D), the clinicopathological features for the patients at poor + moderate grade or well grade were described in Table S1. Next, we classified the 919 patients (patients lost follow-up information were excluded) into cytoplasmic YAP1-low and cytoplasmic YAP1-high subgroups by the optimal cut-off value $(\mathrm{H}$-score= 202.5) determined by the maxstat $\mathrm{R}$ package, the results showed there were significant differences between cytoplasmic YAP1-low and cytoplasmic YAP1-high subgroups with respect to the number of resected lymph nodes and differentiation grade. (all $P<0.001$; Table 1). We also classified the 919 patients into YAP1 NCR-low and YAP1 NCR-high subgroups by the optimal cut-off value $(\mathrm{NCR}=0.0482)$ determined by the maxstat $\mathrm{R}$ package, the results showed a significant difference between the YAP1 NCR-low and YAP1 NCR-high subgroups in the TNM stage $(\mathrm{P}=0.02$; Table S2). The above results revealed that high cytoplasmic YAP1 expression may be involved in the aggressiveness of CRC.

\section{High cytoplasmic YAP1 expression is associated with a worse survival in CRC patients}

A univariate and multivariate Cox regression analyses (Further evaluation of meaningful prognostic factors in univariate analysis in multivariate analysis) was applied to determined the independence of the prognostic value of YAP1 on the basis of the DFS and DSS of CRC patients, the results showed that high cytoplasmic YAP1 expression was an independent risk factor of DFS $(\mathrm{HR}=3.255 ; 95 \% \mathrm{CI}=2.290-4.627 ; P<0.001)$ and $\mathrm{DSS}(\mathrm{HR}=4.049 ; 95 \% \mathrm{CI}=2.400$ 6.830; $P<0.001$ ) for CRC patients (Table 2), likewise, low YAP1 NCR was an independent risk factor of DFS $(\mathrm{HR}=2.295 ; 95 \% \mathrm{CI}=1.118-4.711 ; P=0.024)$ and $\mathrm{DSS}(\mathrm{HR}=2.873 ; 95 \% \mathrm{CI}=1.045$ 7.902; $P=0.041$ ) for $\mathrm{CRC}$ patients (Table 2), but the univariate Cox regression analysis showed that nuclear YAP1 expression level was not a meaningful prognostic factor either for DFS 
$199(\mathrm{HR}=0.684 ; 95 \% \mathrm{CI}=0.453-1.031 ; P=0.07)$ or $\mathrm{DSS}(\mathrm{HR}=0.860 ; 95 \% \mathrm{CI}=0.412-1.975 ; P=0.688)$

200 for CRC patients (Table 2). Kaplan-Meier analyses with log-rank tests showed that DFS and

201 DSS in the cytoplasmic YAP1-high subgroup were significantly shorter than the cytoplasmic

202 YAP1-low subgroup (all $P<0.001$; Fig. 2A, B), moreover, cytoplasmic YAP1-high subgroups

203 were consistently had shorter DFS and DSS than cytoplasmic YAP1-low subgroups in stage I,

204 II, or III CRC patients respectively (all $P<0.01$; Fig. 2B-D, F-H). We also found DFS and DSS

205 were significantly lower in YAP1 NCR-low subgroup than YAP1 NCR-high subgroup (all

$206 P<0.01$; Fig. S2A). However, there is no significant differences between nuclear YAP1-high

207 subgroup and nuclear YAP1-low subgroup in Kaplan-Meier analyses (all $P>0.05$; Fig. S2B).

208 High cytoplasmic YAP1 expression is associated with a worse survival in stage III CRC

209 patients who received chemotherapy

210 To evaluate whether cytoplasmic YAP1 expression level could be an indicator of whether

211 certain patients population could benefit from adjuvant chemotherapy, the stage III patients were

212 divided into two groups respectively (all stage III patients received adjuvant chemotherapy),

213 either did or did not receive adjuvant chemotherapy (Table S3), for stage III patients who

214 received adjuvant chemotherapy, Kaplan-Meier analyses with log-rank tests showed that DFS

215 and DSS in the cytoplasmic YAP1-high subgroup were significantly shorter than the cytoplasmic

216 YAP1-low subgroup (all $P<0.001$; Fig. 3A-B), but there were no significant differences between

217 YAP1-high subgroup and YAP1-low subgroup in DFS and DSS for stage III patients without

218 adjuvant chemotherapy (all $P>0.05$; Fig. 3C-D) . Besides, for stage III patients who received

219 adjuvant chemotherapy, Kaplan-Meier analyses also showed that DFS and DSS in the low YAP1

220 NCR subgroup were significantly shorter than the high YAP1 NCR subgroup (all $P<0.001$; Fig.

221 S3A-B), but there were no significant differences between high YAP1 NCR subgroup and low

222 YAP1 NCR subgroup in DFS and DSS for stage III patients without adjuvant chemotherapy (all

$223 P>0.05$; Fig. S3C-D). Therefore, high cytoplasmic YAP1 expression is associated with a worse

224 survival in stage III CRC patients who received chemotherapy.

\section{Discussion}

226 Primarily, the results in this study showed that in one TCGA dataset and eight GEO

227 datasets, the mRNA expression of YAP1 in CRC tissues was consistently higher in CRC tissues

228 compared with the adjacent normal tissue. Further, the IHC examination of YAP1 confirmed that

229 epithelial cytoplasmic YAP1 protein expression were significantly elevated in CRC tissues 
230 compared with the adjacent normal tissue in the Yunnan Cancer Hospital, and YAP1 NCR

231 gradually decrease in adjacent normal tissues, adenomas, primary cancers, and metastatic CRC.

232 Prior studies had illustrated the expression of YAP1 in a various cancers including CRC (Cottini

233 et al. 2014; Ehsanian et al. 2010; Kang et al. 2011; Kim et al. 2014; Levy et al. 2007; Liu et al.

234 2013; Matallanas et al. 2007; Wang et al. 2013a; Wang et al. 2010; Xu et al. 2009; Yu et al.

235 2013; Yuan et al. 2008), but the association between subcellular localization of YAP1 and

236 aggressiveness of CRC has been neglected. In this study, the expression pattern of YAP1 in the

237 Yunnan Cancer Hospital cohort reveal that the increased cytoplasmic YAP1 expression may be

238 associated with the progression of CRC.

239 The analysis of association between YAP1 expression and CRC patients'

240 clinicopathological features showed that cytoplasmic YAP1 expression was related to

241 differentiation grade and YAP1 NCR was related to TNM stage. Further, CRC patients were

242 divided into cytoplasmic-high YAP1 and cytoplasmic-low YAP1 subgroups by the optimal cut-

243 off value $(\mathrm{H}$-score=202.5), meanwhile, classify CRC patients into YAP1 NCR-low and YAP1

244 NCR-high subgroups according to the optimal cut-off value (NCR=0.0482). We found that DFS

245 and DSS in the cytoplasmic YAP1-high subgroup were significantly shorter than the cytoplasmic

246 YAP1-low subgroup, and DFS and DSS were significantly lower in YAP1 NCR-low subgroup

247 than YAP1 NCR-high subgroup. High cytoplasmic YAP1 expression and low YAP1 NCR were

248 found to be independent risk factors for CRC prognosis in multivariate Cox analysis (after

249 correcting confounding variables), above results indicated that cytoplasmic YAP1 may be used

250 as a indicator for staging of tumor. This is the first study to show the potential association

251 between subcellular localization of YAP1 and CRC patients' clinicopathological characteristics.

252 Adjuvant chemotherapy (FOLFOX/CapeOX regimen) is currently the most effective

253 cytotoxic regimen for the treatment of CRC, FOLFOX adjuvant therapy can significant improve

254 the survival of CRC patients (Gustavsson et al. 2015). However, adjuvant chemotherapy also has

255 some side effects, such as myelotoxicity, neurotoxicity or gastrointestinal toxicity which can be

256 fatal and cause complications (Mohelnikova-Duchonova et al. 2014), therefore, biomarkers

257 predicting the benefit of chemotherapy are urgently needed. Our study clearly demonstrated that

258 high cytoplasmic YAP1 expression is associated with a worse survival in stage III CRC patients

259 who received chemotherapy. Currently, microsatellite instability (MSI) is the only effective

260 indicator for prognosis and suitable chemotherapy regime for colorectal cancer patients

261 (Hemminki et al. 2000; Popat et al. 2005), therefore, a new biomarker is urgently needed to

262 instruct us to determine if a population is suitable for adjuvant chemotherapy. Therefore,

263 cytoplasmic YAP1 may have crucial clinical implications and deserve further study. 
264 There is some evidence to suggest that YAP1 is retained in the cytoplasm by AKT 265 phosphorylation (Basu et al. 2003) or through binding LATS1 (Matallanas et al. 2007), and

266 YAP1 functions as an oncogene which can promote CRC progression by activating the

267 ERK/PI3K-AKT signaling pathway (Wang et al. 2017; Zhang et al. 2016). And LATS1/2 has

268 been reported have a suppress role in cancer immunity(Moroishi et al. 2016), and this

269 phenomenon may be a reason why YAP1 cytoplasmic localization is associate with the

270 progression and poor prognosis of CRC. In another way, YAP1 acts as a tumor suppressor gene

271 interacting with p73 to cause transcription of proapoptotic gene puma (Matallanas et al. 2007),

272 but the apoptosis can be suppressed by enhancing the retention of YAP1 in cytoplasm. This may

273 be the reason why high cytoplasmic YAP1 expression and low YAP1 NCR is associated with the

274 progression and poor prognosis of CRC. Recent research has suggested that upregulation of

275 EGFR by YAP1 has contributed to confer chemoresistance to esophageal cancer cells (Song et

276 al. 2015), another study suggested that YAP1 confers Colon cancer cells chemoresistance to

277 5FU chemotherapy (Touil et al. 2014), Therefore, YAP1 may promote CRC progression, high

278 cytoplasmic YAP1 expression is associated with a worse survival in stage III CRC patients who

279 received chemotherapy. However, the suggestions above are speculative, further mechanistic

280 studies are required to explain the results.

\section{Conclusion}

282 In this study, we provided important evidence that increased cytoplasmic YAP1 correlated 283 with the malignant phenotype in CRC. Importantly, the results show that increased cytoplasmic

284 YAP1 was significantly associated with poor prognosis in CRC patients. More importantly, high

285 cytoplasmic YAP1 expression is associated with a worse survival in stage III CRC patients who

286 received chemotherapy. Our study has revealed that Cytoplasmic YAP1 could be utilized as

287 prognostic factors in CRC patients and may be indicators of whether a certain patient population

288 could benefit from postoperative chemotherapy, however, the molecular mechanisms behind it

289 remain unknown and need to be further investigated.

290 Acknowledgments

291 This work was supported by the grants from the National Natural Science Foundation of P. R.

292 China (No. 81460435 and No. 81860494 to Shangyong Zheng).

293 Disclosure 
294 The authors have no conflicts of interest in this work.

\section{References}

296

297

298

299

300

301

302

303

304

305

306

307

308

309

310

311

312

313

314

315

316

317

318

319

320

321

322

323

324

325

326

327

328

329

330

331

Basu S, Totty NF, Irwin MS, Sudol M, and Downward J. 2003. Akt Phosphorylates the YesAssociated Protein, YAP, to Induce Interaction with 14-3-3 and Attenuation of p73Mediated Apoptosis. Molecular Cell 11:11-23.

Binefa G, Rodriguez-Moranta F, Teule A, and Medina-Hayas M. 2014. Colorectal cancer: from prevention to personalized medicine. World J Gastroenterol 20:6786-6808.

Cottini F, Hideshima T, Xu C, Sattler M, Dori M, Agnelli L, ten Hacken E, Bertilaccio MT, Antonini E, Neri A, Ponzoni M, Marcatti M, Richardson PG, Carrasco R, Kimmelman AC, Wong KK, Caligaris-Cappio F, Blandino G, Kuehl WM, Anderson KC, and Tonon G. 2014. Rescue of Hippo coactivator YAP1 triggers DNA damage-induced apoptosis in hematological cancers. Nat Med 20:599-606.

Detre S, Saclani Jotti G, and Dowsett M. 1995. A "quickscore" method for immunohistochemical semiquantitation: validation for oestrogen receptor in breast carcinomas. J Clin Pathol 48:876-878.

Ehsanian R, Brown M, Lu H, Yang XP, Pattatheyil A, Yan B, Duggal P, Chuang R, Doondeea J, Feller S, Sudol M, Chen Z, and Van Waes C. 2010. YAP dysregulation by phosphorylation or DeltaNp63-mediated gene repression promotes proliferation, survival and migration in head and neck cancer subsets. Oncogene 29:6160-6171.

Fisher KW, Lopez-Beltran A, Montironi R, and Cheng L. 2015. Precision medicine in colorectal cancer: evolving genomic landscape and emerging consensus. Future Oncol.

Garcia HH, Brar GA, Nguyen DH, Bjeldanes LF, and Firestone GL. 2005. Indole-3-Carbinol (I3C) Inhibits Cyclin-dependent Kinase-2 Function in Human Breast Cancer Cells by Regulating the Size Distribution, Associated Cyclin E Forms, and Subcellular Localization of the CDK2 Protein Complex. Journal of Biological Chemistry 280:8756-8764.

Gustavsson B, Carlsson G, Machover D, Petrelli N, Roth A, Schmoll HJ, Tveit KM, and Gibson F. 2015. A review of the evolution of systemic chemotherapy in the management of colorectal cancer. Clin Colorectal Cancer 14:1-10.

Hardingham JE, Grover P, Winter M, Hewett PJ, Price TJ, and Thierry B. 2015. Detection and Clinical Significance of Circulating Tumor Cells in Colorectal Cancer--20 Years of Progress. Mol Med 21 Suppl 1:S25-31.

Hemminki A, Mecklin JP, Jarvinen H, Aaltonen LA, and Joensuu H. 2000. Microsatellite instability is a favorable prognostic indicator in patients with colorectal cancer receiving chemotherapy. Gastroenterology 119:921-928.

Justice RW, Zilian O, Woods DF, Noll M, and Bryant PJ. 1995. The Drosophila tumor suppressor gene warts encodes a homolog of human myotonic dystrophy kinase and is required for the control of cell shape and proliferation. Genes Dev 9:534-546.

Kang W, Tong JH, Chan AW, Lee TL, Lung RW, Leung PP, So KK, Wu K, Fan D, Yu J, Sung

PeerJ reviewing PDF | (2020:05:49163:2:0:NEW 25 Oct 2020) 
332

333

334

335

336

337

338

339

340

341

342

343

344

345

346

347

348

349

350

351

352

353

354

355

356

357

358

359

360

361

362

363

364

365

366

367

368

369

370

JJ, and To KF. 2011. Yes-associated protein 1 exhibits oncogenic property in gastric cancer and its nuclear accumulation associates with poor prognosis. Clin Cancer Res 17:21302139.

Kim SK, Jung WH, and Koo JS. 2014. Yes-associated protein (YAP) is differentially expressed in tumor and stroma according to the molecular subtype of breast cancer. Int J Clin Exp Pathol 7:3224-3234.

Levy D, Adamovich Y, Reuven N, and Shaul Y. 2007. The Yes-associated protein 1 stabilizes p73 by preventing Itch-mediated ubiquitination of p73. Cell Death Differ 14:743-751.

Liu JY, Li YH, Lin HX, Liao YJ, Mai SJ, Liu ZW, Zhang ZL, Jiang LJ, Zhang JX, Kung HF, Zeng YX, Zhou FJ, and Xie D. 2013. Overexpression of YAP 1 contributes to progressive features and poor prognosis of human urothelial carcinoma of the bladder. BMC Cancer 13:349.

Lobo GP, Waite KA, Planchon SM, Romigh T, Nassif NT, and Eng C. 2009. Germline and somatic cancer-associated mutations in the ATP-binding motifs of PTEN influence its subcellular localization and tumor suppressive function. Hum Mol Genet 18:2851-2862.

Marin JJ, Sanchez de Medina F, Castano B, Bujanda L, Romero MR, Martinez-Augustin O, MoralAvila RD, and Briz O. 2012. Chemoprevention, chemotherapy, and chemoresistance in colorectal cancer. Drug Metab Rev 44:148-172.

Matallanas D, Romano D, Yee K, Meissl K, Kucerova L, Piazzolla D, Baccarini M, Vass JK, Kolch W, and O'Neill E. 2007. RASSF1A elicits apoptosis through an MST2 pathway directing proapoptotic transcription by the 73 tumor suppressor protein. Mol Cell 27:962975.

Mohelnikova-Duchonova B, Melichar B, and Soucek P. 2014. FOLFOX/FOLFIRI pharmacogenetics: the call for a personalized approach in colorectal cancer therapy. World J Gastroenterol 20:10316-10330.

Moroishi T, Hayashi T, Pan WW, Fujita Y, Holt MV, Qin J, Carson DA, and Guan KL. 2016. The Hippo Pathway Kinases LATS1/2 Suppress Cancer Immunity. Cell 167:1525-1539 e1517.

Nagtegaal ID, Quirke P, and Schmoll HJ. 2012. Has the new TNM classification for colorectal cancer improved care. Nature Reviews Clinical Oncology 9:119-123.

Pan Y, Cao F, Guo A, Chang W, Chen X, Ma W, Gao X, Guo S, Fu C, and Zhu J. 2015. Endoplasmic reticulum ribosome-binding protein 1, RRBP1, promotes progression of colorectal cancer and predicts an unfavourable prognosis. Br J Cancer 113:763-772.

Popat S, Hubner R, and Houlston RS. 2005. Systematic review of microsatellite instability and colorectal cancer prognosis. J Clin Oncol 23:609-618.

Song S, Honjo S, Jin J, Chang SS, Scott AW, Chen Q, Kalhor N, Correa AM, Hofstetter WL, Albarracin CT, Wu TT, Johnson RL, Hung MC, and Ajani JA. 2015. The Hippo Coactivator YAP1 Mediates EGFR Overexpression and Confers Chemoresistance in Esophageal Cancer. Clin Cancer Res 21:2580-2590.

Torre LA, Bray F, Siegel RL, Ferlay J, Lortet-Tieulent J, and Jemal A. 2015. Global cancer 
371

372

373

374

375

376

377

378

379

380

381

382

383

384

385

386

387

388

389

390

391

392

393

394

395

396

397

398

399

400

401

402

403

404

405

406

407

408

409

statistics, 2012. CA Cancer J Clin 65:87-108.

Touil Y, Igoudjil W, Corvaisier M, Dessein AF, Vandomme J, Monte D, Stechly L, Skrypek N, Langlois C, Grard G, Millet G, Leteurtre E, Dumont P, Truant S, Pruvot FR, Hebbar M, Fan F, Ellis LM, Formstecher P, Van Seuningen I, Gespach C, Polakowska R, and Huet G. 2014. Colon cancer cells escape 5FU chemotherapy-induced cell death by entering stemness and quiescence associated with the c-Yes/YAP axis. Clin Cancer Res 20:837846.

Vaquero J, Nguyen Ho-Bouldoires TH, Claperon A, and Fouassier L. 2017. Role of the PDZscaffold protein NHERF1/EBP50 in cancer biology: from signaling regulation to clinical relevance. Oncogene 36:3067-3079.

Wang C, Gu C, Jeong KJ, Zhang D, Guo W, Lu Y, Ju Z, Panupinthu N, Yang JY, Gagea MM, Ng PK, Zhang F, and Mills GB. 2017. YAP/TAZ-Mediated Upregulation of GAB2 Leads to Increased Sensitivity to Growth Factor-Induced Activation of the PI3K Pathway. Cancer Res 77:1637-1648.

Wang L, Shi S, Guo Z, Zhang X, Han S, Yang A, Wen W, and Zhu Q. 2013a. Overexpression of YAP and TAZ is an independent predictor of prognosis in colorectal cancer and related to the proliferation and metastasis of colon cancer cells. PLoS One 8:e65539.

Wang Y, Dong Q, Zhang Q, Li Z, Wang E, and Qiu X. 2010. Overexpression of yes-associated protein contributes to progression and poor prognosis of non-small-cell lung cancer. Cancer Sci 101:1279-1285.

Wang Y, Xie C, Li Q, Xu K, and Wang E. 2013b. Clinical and prognostic significance of Yesassociated protein in colorectal cancer. Tumour Biol 34:2169-2174.

Xu MZ, Yao TJ, Lee NP, Ng IO, Chan YT, Zender L, Lowe SW, Poon RT, and Luk JM. 2009. Yes-associated protein is an independent prognostic marker in hepatocellular carcinoma. Cancer 115:4576-4585.

Yang R, Cai TT, Wu XJ, Liu YN, He J, Zhang XS, Ma G, and Li J. 2018. Tumor YAP1 and PTEN expression correlates with tumor-associated myeloid suppressor cell expansion and reduced survival in colorectal cancer. Immunology.

Yu SJ, Hu JY, Kuang XY, Luo JM, Hou YF, Di GH, Wu J, Shen ZZ, Song HY, and Shao ZM. 2013. MicroRNA-200a Promotes Anoikis Resistance and Metastasis by Targeting YAP1 in Human Breast Cancer. Clinical Cancer Research 19:1389-1399.

Yuan M, Tomlinson V, Lara R, Holliday D, Chelala C, Harada T, Gangeswaran R, Manson-Bishop C, Smith P, Danovi SA, Pardo O, Crook T, Mein CA, Lemoine NR, Jones LJ, and Basu S. 2008. Yes-associated protein (YAP) functions as a tumor suppressor in breast. Cell Death Differ 15:1752-1759.

Zeng ZL, Luo HY, Yang J, Wu WJ, Chen DL, Huang P, and Xu RH. 2014. Overexpression of the circadian clock gene Bmall increases sensitivity to oxaliplatin in colorectal cancer. Clin Cancer Res 20:1042-1052.

Zhang Y, Yuan J, Zhang X, Yan F, Huang M, Wang T, Zheng X, and Zhang M. 2016. Angiomotin 
410 promotes the malignant potential of colon cancer cells by activating the YAP-ERK/PI3K-

411 AKT signaling pathway. Oncol Rep 36:3619-3626.

412 
413 Figure legends

414 Fig. 1 Differences in YAP1 expression between CRC tissues and adjacent normal tissues.

415 (A-D) Bioinformatics analyses of YAP1 mRNA expression between cancer and cancer related 416 specimens in one TCGA dataset and three GEO datasets. (E) Comparison of YAP1 expression

417 level among different colorectal pathological tissues by cytoplasmic YAP1 H-score, (F) nuclear

418 YAP1 H-score, or (G) YAP1 NCR H-score. (H-M) Representative YAP1 staining in normal

419 tissues and cancer tissues, the blue staining represents the nuclear staining and the brown

420 staining represents the YAP1 positive staining, cancer tissue have the higher cytoplasmic YAP1

421 H-score, higher nuclear YAP1 H-score and lower NCR than normal tissue, scale bars: $100 \mu \mathrm{m}$.

$422 * P<0.05 ; * * P<0.01 ; * * * P<0.001 ; * * * * P<0.0001$; ns, no significance.

423 Fig. 2 High cytoplasmic YAP1 expression is associated with a worse survival in CRC 424 patients.

425 (A-D) Associations between cytoplasmic YAP1 expression and DFS in the patient subgroups

426 with different stage. (E-H) Associations between cytoplasmic YAP1 expression and DSS in the

427 patient subgroups with different stage. Patients with stages I - III, stage I, stage II, or stage III

428 were dichotomized into the cytoplasmic YAP1-high subgroups and cytoplasmic YAP1-low

429 subgroups according to optimal cut-off value. Kaplan-Meier survival curves reveal DFS and

430 DSS in patients with each TNM stage CRC. $P$-values are from Kaplan-Meier analysis with log431 rank test.

432 Fig. 3 Adjuvant chemotherapy has a differential effect on patients with different 433 cytoplasmic YAP1 expression levels.

434 Associations between cytoplasmic YAP1 expression and DFS (A) or DSS (B) in the stage III 435 patients with chemotherapy. Associations between cytoplasmic YAP1 expression and DFS (C)

436 or DSS (D) in the stage III patients without chemotherapy. $P$-values are from Kaplan-Meier 437 analysis with log-rank test.

438 Fig. S1 Supplementary bioinformatics analyses and differences in YAP1 expression 439 between different differentiation grades.

440 (A) Bioinformatics analyses of YAP1 mRNA expression levels in cancer and cancer-related 441 specimens in five GEO datasets. (B) Correlation scatter plot of Cytoplasmic vs Nuclear YAP1

442 H-score. (C) Comparison of YAP1 expression levels between different differentiation grades by 443 cytoplasmic YAP1 H-score (D) nuclear YAP1 H-score, or (E) YAP1 NCR H-score. *P<0.05; $444 * * \mathrm{P}<0.01 ; * * * \mathrm{P}<0.001 ; * * * * \mathrm{P}<0.0001 ;$ ns, no significance. 
445 Fig. S2 Low YAP1 NCR is associated with a worse survival in CRC patients.

446 (A) Associations between nuclear YAP1 expression and DFS or DSS in patients with stages I -

447 III. Patients with stages I - III were dichotomized into nuclear YAP1-high subgroups and nuclear

448 YAP1-low subgroups according to optimal cut-off value. (B) Associations between YAP1 NCR

449 and DFS or DSS in patients with stages I - III. Patients with stages I - III were dichotomized

450 into YAP1 NCR-high subgroups and YAP1 NCR-low subgroups according to optimal cut-off

451 value. $P$-values are from Kaplan-Meier analysis with log-rank test.

452 Fig. S3 Adjuvant chemotherapy has a differential effect on patients with different YAP1

453 NCR.

454 Associations between YAP1 NCR and DFS (A) or DSS (B) in the stage III patients with

455 chemotherapy. Associations betweenYAP1 NCR and DFS (C) or DSS (D) in the stage III

456 patients without chemotherapy. $P$-values are from Kaplan-Meier analysis with log-rank test. 457 


\section{Figure 1}

Differences in YAP1 expression between CRC tissues and adjacent normal tissues.

(A-D) Bioinformatics analyses of YAP1 mRNA expression between cancer and cancer related specimens in one TCGA dataset and three GEO datasets. (E) Comparison of YAP1 expression level among different colorectal pathological tissues by cytoplasmic YAP1 H-score, (F) nuclear YAP1 H-score, or (G) YAP1 NCR H-score. (H-M) Representative YAP1 staining in normal tissues and cancer tissues, the blue staining represents the nuclear staining and the brown staining represents the YAP1 positive staining, cancer tissue have the higher cytoplasmic YAP1 H-score, higher nuclear YAP1 H-score and lower NCR than normal tissue, scale bars: $100 \mu$ m. $* P<0.05 ;{ }^{* * P}<0.01 ;{ }^{* * * P}<0.001 ; * * * P<0.0001 ;$ ns, no significance. 

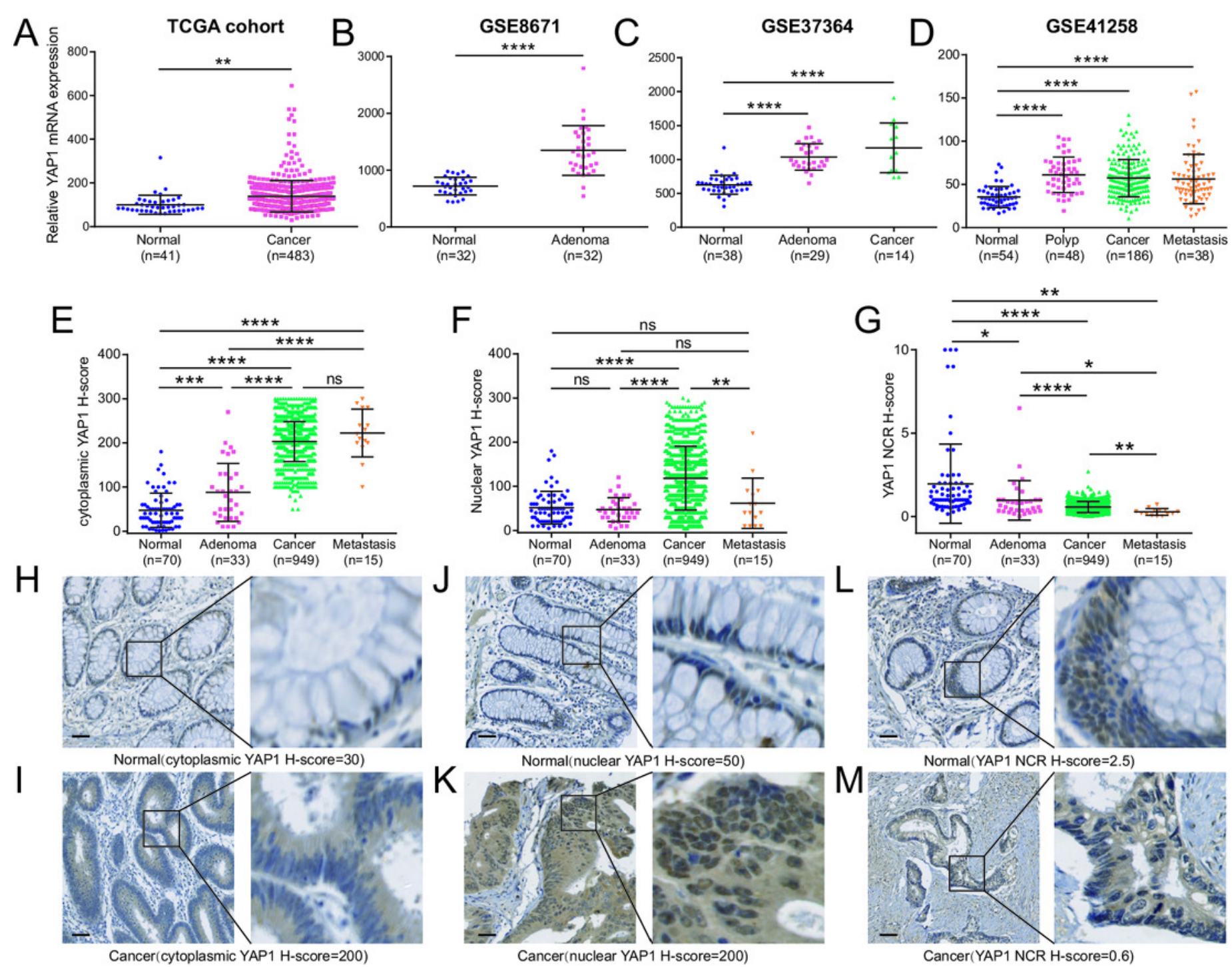


\section{Figure 2}

High cytoplasmic YAP1 expression is associated with worse survival in CRC patients.

(A-D) Associations between cytoplasmic YAP1 expression and DFS in the patient subgroups with different stage. (E-H) Associations between cytoplasmic YAP1 expression and DSS in the patient subgroups with different stage. Patients with stages I-III, stage I, stage II, or stage III were dichotomized into the cytoplasmic YAP1-high subgroups and cytoplasmic YAP1-low subgroups according to optimal cut-off value. Kaplan-Meier survival curves reveal DFS and DSS in patients with each TNM stage CRC. $P$-values are from Kaplan-Meier analysis with logrank test.

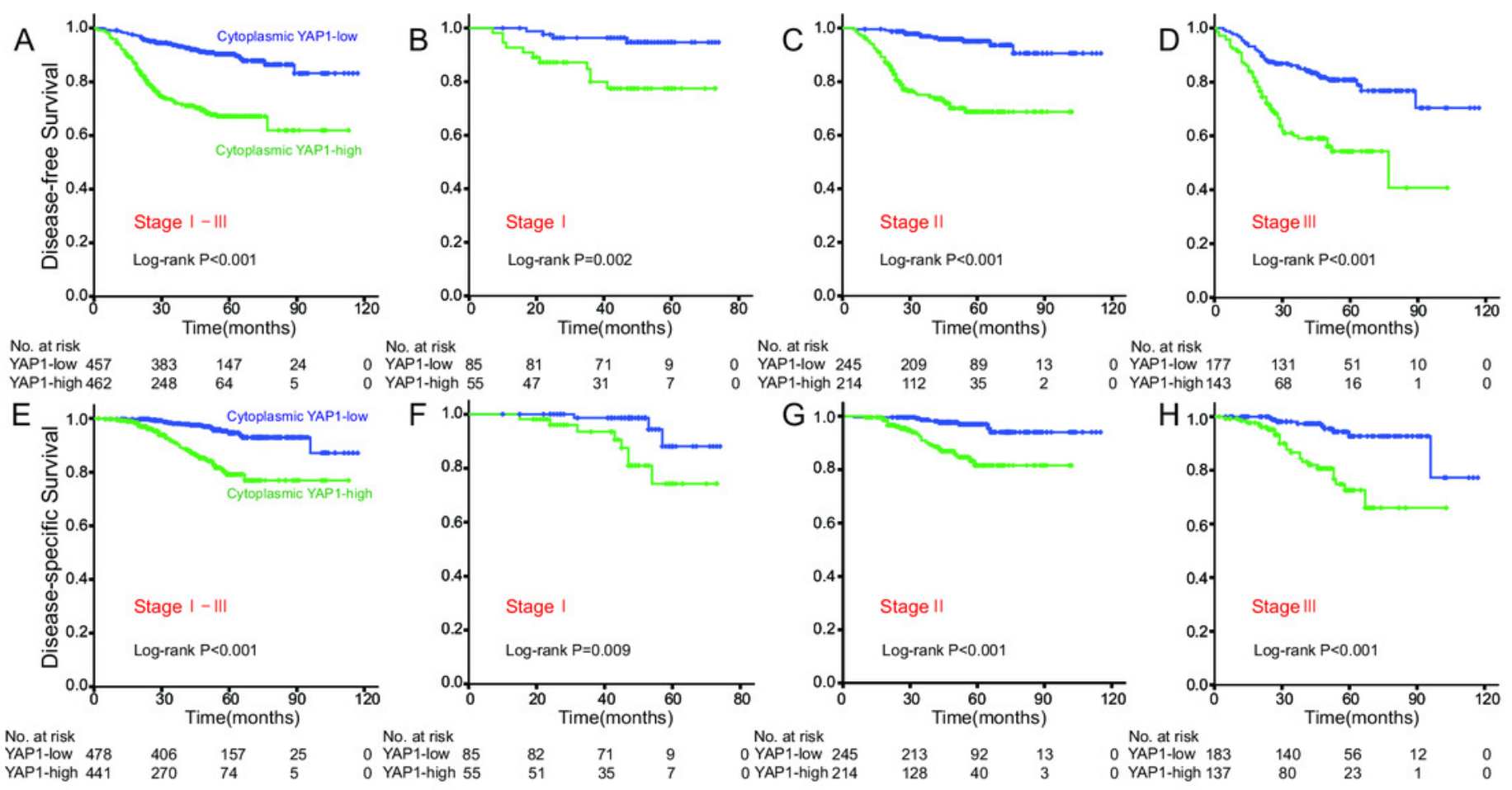




\section{Figure 3}

Adjuvant chemotherapy has a differential effect on patients with different cytoplasmic YAP1 expression levels.

Associations between cytoplasmic YAP1 expression and DFS (A) or DSS (B) in the stage III patients with chemotherapy. Associations between cytoplasmic YAP1 expression and DFS (C) or DSS (D) in the stage III patients without chemotherapy. $P$-values are from Kaplan-Meier analysis with log-rank test. 


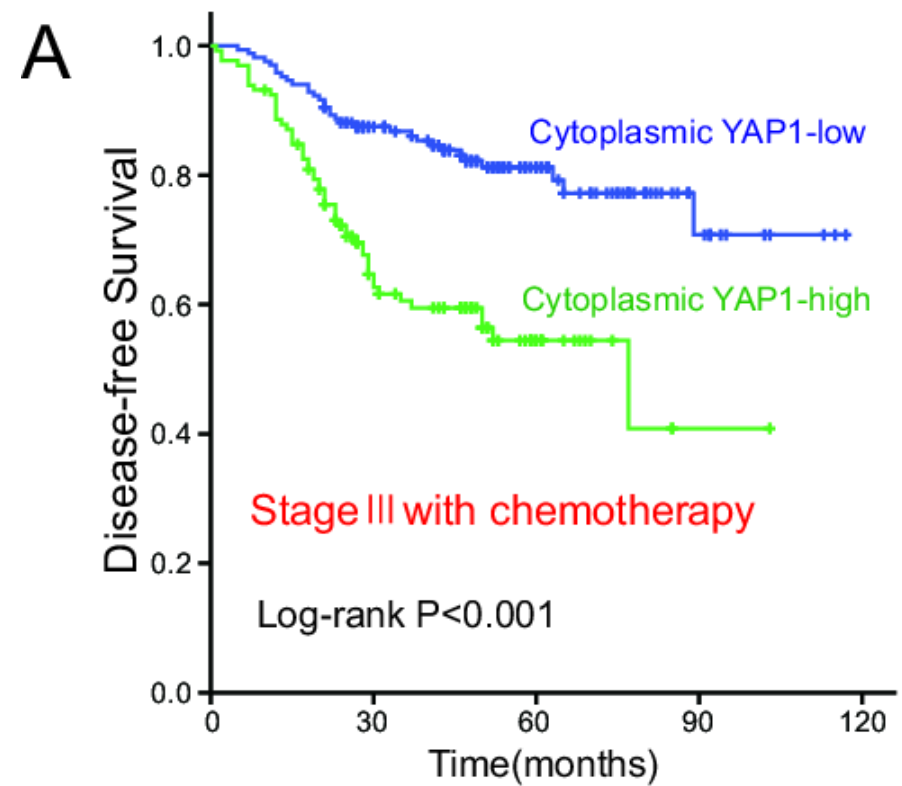

No. at risk

YAP1-low 169

YAP1-high 132

128

63
B

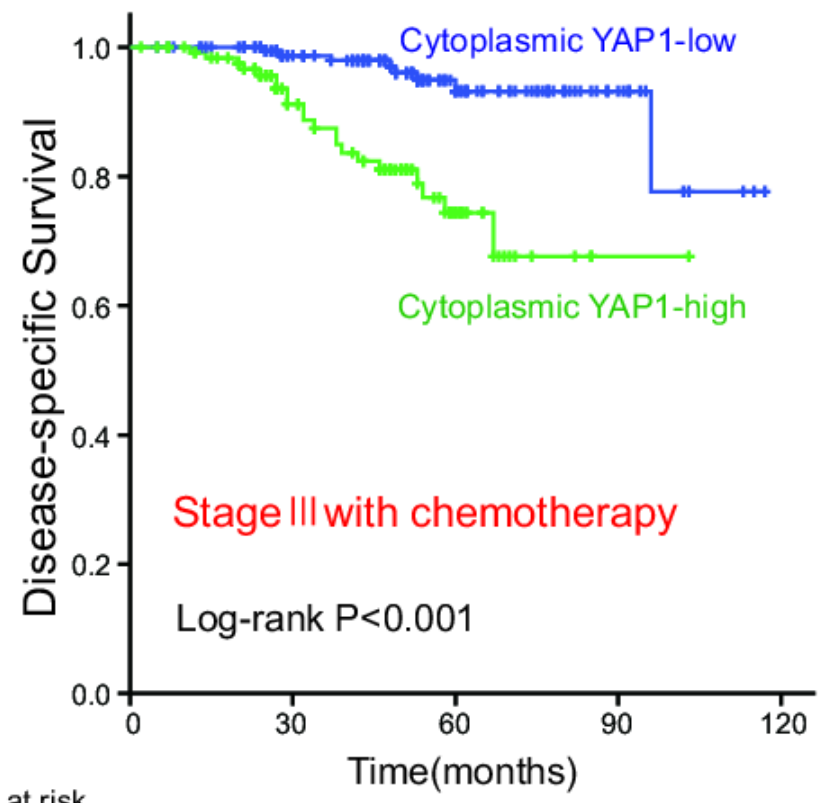

No. at risk

YAP1-low 175

YAP1-high 126

$\begin{array}{cccc}137 & 55 & 12 & 0 \\ 74 & 23 & 1 & 0\end{array}$

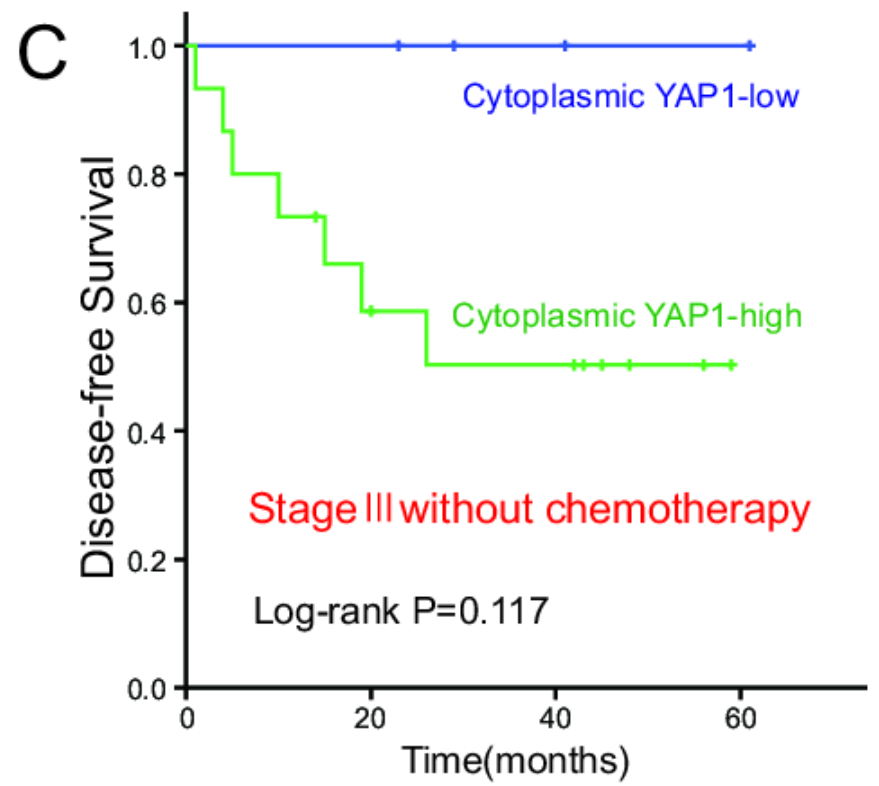

$\begin{array}{lcccc}\text { No. at risk } & & & & \\ \text { YAP1-low } & 4 & 2 & 1 & 0 \\ \text { YAP1-high } & 15 & 7 & 5 & 0\end{array}$
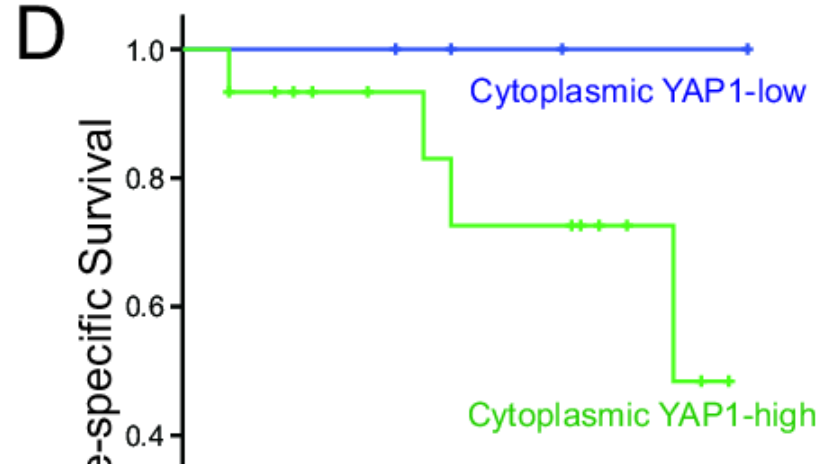

Stage III without chemotherapy

Log-rank $P=0.253$

No. at risk

$\begin{array}{lclll}\text { YAP1-low } & 4 & 3 & 1 & 0 \\ \text { YAP1-high } & 15 & 9 & 6 & 0\end{array}$ 


\section{Table 1 (on next page)}

Associations of cytoplasmic YAP1 expression with demographic and clinical variables of 919 CRC patients

Notes: ${ }^{*} \chi 2$ test. ${ }^{* *}$ Student t-test. ${ }^{* * *}$ Mann-Whitney $U$ test (non-parametric). Missing values are excluded for all statistic tests. Abbreviations: YAP1, Yes associated protein 1; TNM, tumor-node-metastasis; CEA, carcinoembryonic antigen; CA19-9, carbohydrate antigen 19-9. 
1 Table 1. Associations of cytoplasmic YAP1 expression with demographic and clinical 2 variables of 919 CRC patients

\begin{tabular}{|c|c|c|c|c|}
\hline \multirow[t]{2}{*}{ Characteristics } & \multirow{2}{*}{$\begin{array}{c}\text { Total } \\
(n=919)\end{array}$} & \multicolumn{2}{|c|}{$\begin{array}{c}\text { Cytoplasmic YAP1 } \\
\text { expression level }\end{array}$} & \multirow[t]{2}{*}{$P$ Value ${ }^{*}$} \\
\hline & & $\operatorname{Low}(n=457)$ & $\operatorname{High}(n=462)$ & \\
\hline Mean age \pm SD(year) & $60.1 \pm 12.4$ & $61.4 \pm 12.3$ & $60.7 \pm 12.5$ & $0.389^{* *}$ \\
\hline $\operatorname{Sex}(n(\%))$ & & & & 0.686 \\
\hline Men & $549(59.7)$ & $270(59.1)$ & $279(60.4)$ & \\
\hline Women & $370(40.3)$ & $187(40.9)$ & $183(39.6)$ & \\
\hline Disease location (n(\%)) & & & & 0.632 \\
\hline Rectum & $512(55.7)$ & $251(54.9)$ & $261(56.5)$ & \\
\hline Colon & $407(44.3)$ & $206(45.1)$ & $201(43.5)$ & \\
\hline Differentiation grade (n(\%)) & & & & $<0.001^{* * *}$ \\
\hline Well & $95(10.3)$ & $67(14.7)$ & $28(6.1)$ & \\
\hline Moderately & $779(84.8)$ & $369(80.7)$ & $410(88.7)$ & \\
\hline Poorly & $35(3.8)$ & $14(3.1)$ & $21(4.5)$ & \\
\hline Missing & $10(1.1)$ & $7(1.5)$ & $3(0.6)$ & \\
\hline Resected lymph nodes (n(\%)) & & & & $<0.001$ \\
\hline$<12$ & 201(21.9) & $140(30.6)$ & $61(13.2)$ & \\
\hline$\geq 12$ & $718(78.1)$ & $317(69.4)$ & $401(86.8)$ & \\
\hline TNM stage (n(\%)) & & & & $0.362^{* * *}$ \\
\hline I & $140(15.2)$ & $65(14.2)$ & $75(16.2)$ & \\
\hline II & $459(49.9)$ & $245(53.6)$ & $214(46.3)$ & \\
\hline III & $320(34.8)$ & $147(32.2)$ & $173(37.4)$ & \\
\hline Chemotherapy (n(\%)) & & & & 0.730 \\
\hline Yes & $671(73.0)$ & $336(73.5)$ & $335(72.5)$ & \\
\hline No & $248(27.0)$ & $121(26.5)$ & $127(27.5)$ & \\
\hline Serum CEA (n(\%)) & & & & 0.451 \\
\hline$<5 \mathrm{ng} / \mathrm{ml}$ & $568(61.8)$ & $288(63)$ & $280(60.6)$ & \\
\hline$\geq 5 \mathrm{ng} / \mathrm{ml}$ & $351(38.2)$ & $169(37)$ & $182(39.4)$ & \\
\hline Serum CA19-9 (n(\%)) & & & & 0.686 \\
\hline$<37 \mathrm{U} / \mathrm{ml}$ & $788(85.7)$ & $394(86.2)$ & $394(85.3)$ & \\
\hline$\geq 37 \mathrm{U} / \mathrm{ml}$ & $131(14.3)$ & $63(13.8)$ & $68(14.7)$ & \\
\hline
\end{tabular}

3 Notes: ${ }^{*} \chi 2$ test.

$4 \quad{ }^{* *}$ Student t-test.

$5{ }^{* * *}$ Mann-Whitney U test (non-parametric). Missing values are excluded for all statistic tests.

6 Abbreviations: YAP1, Yes associated protein 1; TNM, tumor-node-metastasis; CEA, carcinoembryonic 7 antigen; CA19-9, carbohydrate antigen 19-9. 


\section{Table 2 (on next page)}

Cox regression analysis of immunohistochemistry YAP1 expression and clinicopathological covariates in patients with CRC

Abbreviations: $\mathrm{HR}$, hazard ratio; $\mathrm{Cl}$, confidence interval; YAP1, Yes associated protein 1;

TNM, tumor-node-metastasis; CEA, carcinoembryonic antigen; CA19-9, carbohydrate antigen 19-9; NCR, Nuclear/Cytoplasmic Ratio. 
Table 2. Cox regression analysis of immunohistochemistry YAP1 expression and clinicopathological covariates in patients with

2

CRC

\begin{tabular}{|c|c|c|c|c|c|c|c|c|}
\hline \multirow{3}{*}{ Characteristics } & \multicolumn{4}{|c|}{ Disease-free Survival } & \multicolumn{4}{|c|}{ Disease-specific Survival } \\
\hline & \multicolumn{2}{|l|}{ Univariate } & \multicolumn{2}{|l|}{ Multivariate } & \multicolumn{2}{|l|}{ Univariate } & \multicolumn{2}{|l|}{ Multivariate } \\
\hline & $\mathrm{HR}(95 \% \mathrm{CI})$ & $\begin{array}{c}P \\
\text { Value }\end{array}$ & $\mathrm{HR}(95 \% \mathrm{CI})$ & $\begin{array}{c}P \\
\text { Value }\end{array}$ & $\mathrm{HR}(95 \% \mathrm{CI})$ & $\begin{array}{c}P \\
\text { Value }\end{array}$ & $\mathrm{HR}(95 \% \mathrm{CI})$ & $\begin{array}{c}P \\
\text { Value }\end{array}$ \\
\hline YAP1-high vs. YAP1-low(cytoplasmic) & $3.891(2.758-5.490)$ & $<0.001$ & $3.255(2.290-4.627)$ & $<0.001$ & $4.291(2.545-7.236)$ & $<0.001$ & $4.049(2.400-6.830)$ & $<0.001$ \\
\hline YAP1-low vs. YAP1-high(NCR) & $2.709(1.331-5.511)$ & 0.006 & $2.295(1.118-4.711)$ & 0.024 & $3.346(1.219-9.183)$ & 0.019 & $2.873(1.045-7.902)$ & 0.041 \\
\hline YAP1-high $v s$. YAP1-low(nuclear) & $0.684(0.453-1.031)$ & 0.070 & & & $0.860(0.412-1.975)$ & 0.688 & & \\
\hline Age $(>=60 v s .<60)$ & $0.897(0.667-1.207)$ & 0.474 & & & $0.891(0.568-1.398)$ & 0.617 & & \\
\hline Sex (female $v s$. male) & $0.867(0.638-1.177)$ & 0.360 & & & $0.817(0.512-1.302)$ & 0.395 & & \\
\hline Location (colon vs. rectum) & $1.068(0.793-1.440)$ & 0.665 & & & $1.159(0.737-1.823)$ & 0.522 & & \\
\hline TNM (per increase in stage) & $1.874(1.474-2.381)$ & $<0.001$ & $1.863(1.471-2.360)$ & $<0.001$ & $1.256(0.889-1.775)$ & 0.196 & & \\
\hline Grade (per increase in grade) & $3.001(1.948-4.625)$ & $<0.001$ & $3.435(2.127-5.548)$ & $<0.001$ & $2.992(1.575-5.685)$ & 0.001 & $2.732(1.383-5.394)$ & 0.004 \\
\hline Chemotherapy (yes vs. no) & $1.705(1.156-2.515)$ & 0.007 & $1.029(0.647-1.637)$ & 0.902 & $1.125(0.662-1.912)$ & 0.663 & & \\
\hline Resected lymph nodes $(\geq 12 v s .<12)$ & $2.675(1.689-4.236)$ & $<0.001$ & $1.780(1.111-2.853)$ & 0.017 & $2.610(1.375-4.954)$ & 0.003 & $1.685(0.874-3.251)$ & 0.120 \\
\hline Serum CEA $(\geq 5$ vs. $<5 \mathrm{ng} / \mathrm{ml})$ & $1.646(1.223-2.215)$ & 0.001 & $1.513(1.120-2.043)$ & 0.007 & $1.378(0.876-2.170)$ & 0.166 & & \\
\hline Serum CA19-9 $(\geq 37$ vs. $<37 \mathrm{U} / \mathrm{ml})$ & $1.766(1.224-2.549)$ & 0.002 & $1.350(0.914-1.995)$ & 0.132 & $1.619(0.906-2.894)$ & 0.104 & & \\
\hline
\end{tabular}

3 Abbreviations: HR, hazard ratio; CI, confidence interval; YAP1, Yes associated protein 1; TNM, tumor-node-metastasis; CEA, carcinoembryonic antigen;

4 CA19-9, carbohydrate antigen 19-9; NCR, Nuclear/Cytoplasmic Ratio. 